$\frac{276}{7-19-19}$ LA-7557-MS

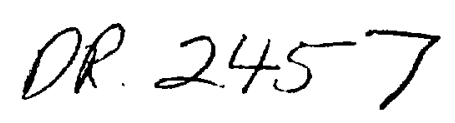

Informal Report

Simplified Multicomponent Phase Transition Model

).

$\frac{\pi}{\frac{0}{0}}$

4

$\underset{\infty}{\infty}$

(1)

MASTER 
LA-7557-MS

Informal Report

UC-79p (Base Technology)

Issued: January 1979

\title{
Simplified Mulłicomponent Phase Transition Model
}

\author{
Ahti J. Suo-Anttila
}
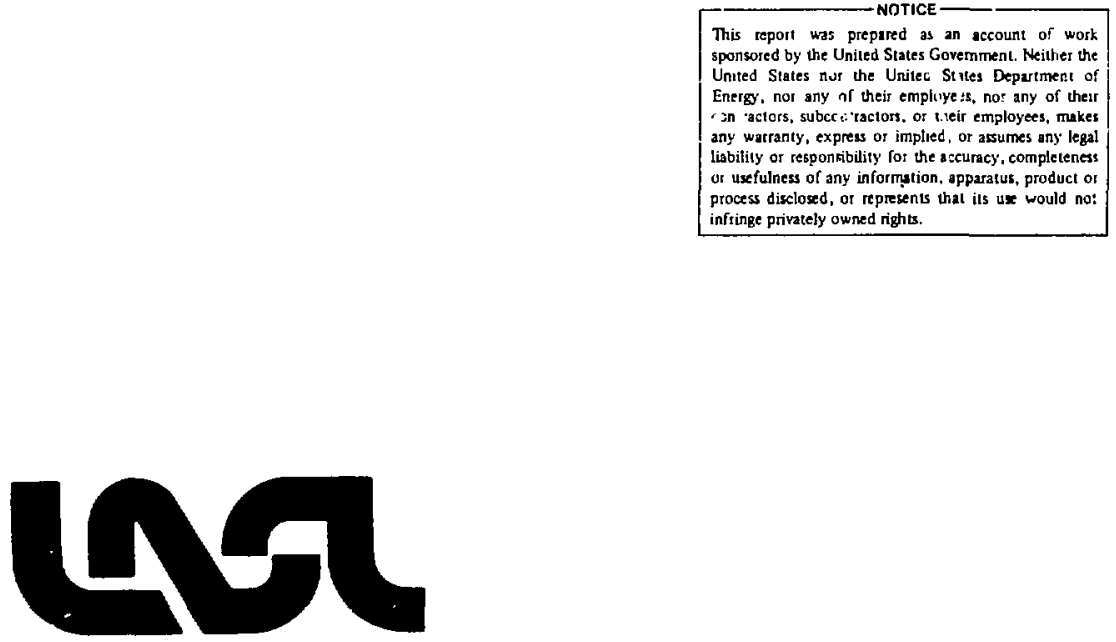


\section{CONTENTS}

Page

ABSTRACT

$\begin{array}{ll}\text { I. INTRODUCTION } & 1\end{array}$

II. THEORY 3

A. The Flux Boundary Conditions 3

B. Thermodynamic Equilibrium of the interface 6

C. The Mass Transfer Coefficient 9

D. The Correction Factor 12

E. Solution Techniques for Simultaneous Heat and Mass Transfer 15

III. MODELING CONCERNS 20

IV. SUMMARY AND CONCLUSIONS 23

$\begin{array}{ll}\checkmark & \text { REFERENCES } \\ & 23\end{array}$ 
by

Anti J. Suo-Anttila

\begin{abstract}
A metnod is presented that will allow one to generate approximate solutions to $\mathrm{N}$ - component phase transition problems. There are $\mathrm{N}-1$ species equations and one energy equation, coupled at the phase transition interface by a thermodynamic relationship. The equivalent "Couette flow" boundary layer equations are utilized to relate the mass and energy fluxes to the overall driving forces. Various simplifying assumptions are made; however, the method is sufficiently general that no simplifying assumptions need be made. A completely rigorous treatment would only introduce additional nonlinearity into the system, rather than change the method.
\end{abstract}

\title{
I. INTRODUCTION
}

Current practice in nuclear reactor safety analysis is to make use of 1 arge computer codes that predict the behavior of a reactor under accident conditions. These codes model a number of physical phenomena among which evaporation and condensation, or simultaneous heat and mass transfer, play an important role. The complexity and variability of accident conditions force one to make use of a general but simple predictive mass transfer theory capable of yielding approximate solutions. The solutions will, in general, have two uses. First, the solutions will usually be of sufficient accuracy for engineering or safety analy'sis purposes. Second, such solutions can serve as an initial step in deciding whether a more refined analysis to the problem is necessary.

The analysis of convective heat and mass transfer is most often confined to the boundary layer. The prediction of transfei rates through the boundary layer normally proceeds in two steps. First, the boundary conditions are specified, and, second, the boundary layer equations are solved subject to some tolerable degree of approximation. 
The boundary conditions are reiatively easy to specify when heat transfer is the only mechanism playing a role. However, when mass transfer is important, the houndary conditions can be quite difficult to specify,especially when multicomponent systems with simultaneous heat transfer and possible chemical reactions are occurring. In this case a simultaneous solution involving heat transfer, thermodynamics and chemical kinetics is involved. For this reason a major portion of the theoretical development in this report will involve setting up the proper boundary conditions for the simultaneous heat and mass transfer prcblem associated with multicomponent systems.

The solutions to the boundary layer equations can take various levels of approximation. First, there are various exact analytical solutions for certzin specified flow fields and geometries. The flow fields and geometries are usually fairly specific and are not applicable to the general case. The next degree of approximation involves the numerical methods. These methods give quite good results but are computationaliy time consuming and require a detailed specification of the flow tield and geometry. In addition, when the flow field is turbulent, useful solutions are very difficult to generate. The last and simplest level of approximation is the development of experimental correlations of the flow field and gecmetry under investigation. The correlations will, when properly evaluated, yield heat and mass transfer conductances (coefficients) which, when combined with the boundary conditions (driving force), yield the rates of transfer. This last method is applicable to both laminar and turbuient flow fields and gives answers usually accurate to within $20-30 \%$, proyided the limits of the correlation have not been exceeded.

Most experimental correlations have been developed for heat transfer, and due to the similarity of the boundary layer equations, mass transfer coefficients can be cierived from the correlations. This analogy between heat and mass transfer is not completely straightforward because in the mass transfer case there is a net flow of material through the boundary layer. This flow of material can be accounted for by a correction factor that forces the mass transfer coefficient to satisfy a simpified form of the boundary layer equations.

The model which is presented hereil is a simplified phase transition model for the following reasons. First, the boundary layer equations are solved by the use of heat and mass transfter coefficients which have been 
corrected to account for material flowing through the boundary layer. Second, the species and energy equations are all coupled at the phase transition interface due to the thermodynamic behavior of the materials involved. Saturation conditions with simplifying assumptions based upon component miscibility are used to govern this interfacial-thermodynamic behavior. Last, a Lewis number equals unity assumption can be used to simplify the nonlinear coupling that orcurs due to the varying thermophysical properties of the materials involved. None of the simplifications mentioned are essential to the model; it is certainly possible to make none of these assumptions and proceed in the same fashion. The major difference would be that more nonlinear coupling would be int. uduced, and this in turn would require more sophisticated and time consuming numerical method's to generate a solution.

\section{THEORY}

A. The Flux Boundary Conditions

The fiux equations can be derived directly from the governing partial differential equations of the boundary layer by integrating and extracting that portion of the equations which represents the mass or energy flux. If mass diffusion due to thermal or pressure gradients is consiciered to be negligible then an expression for the mass flux of species $k$ is ${ }^{1}$

$$
n_{K}=\rho_{K} v_{K}=\rho v m_{K}-\rho D_{K, g} \frac{\partial m_{K}}{\partial y},
$$

where

$$
\begin{aligned}
& n, \rho, v, m, D \quad \begin{array}{l}
\text { are the absolute mass flux, density, velocity, mass } \\
\text { fraction, and mass diffusivity, respectively. }
\end{array}
\end{aligned}
$$

The subscripts $k$ and $g$ imply species $k$ and gas mixture $g$. Additional subscript notation will be introduced as needed to denote pasition within the boundary layer or condensible surface.

- Figure 1 depicts the mass transfer situation. The mass flux $n_{k}$ is defined as being positive into se vapor phase. The subscripts $i$ and o denote the vapor-liquid interface and free stream, respectively. The boundary layer 


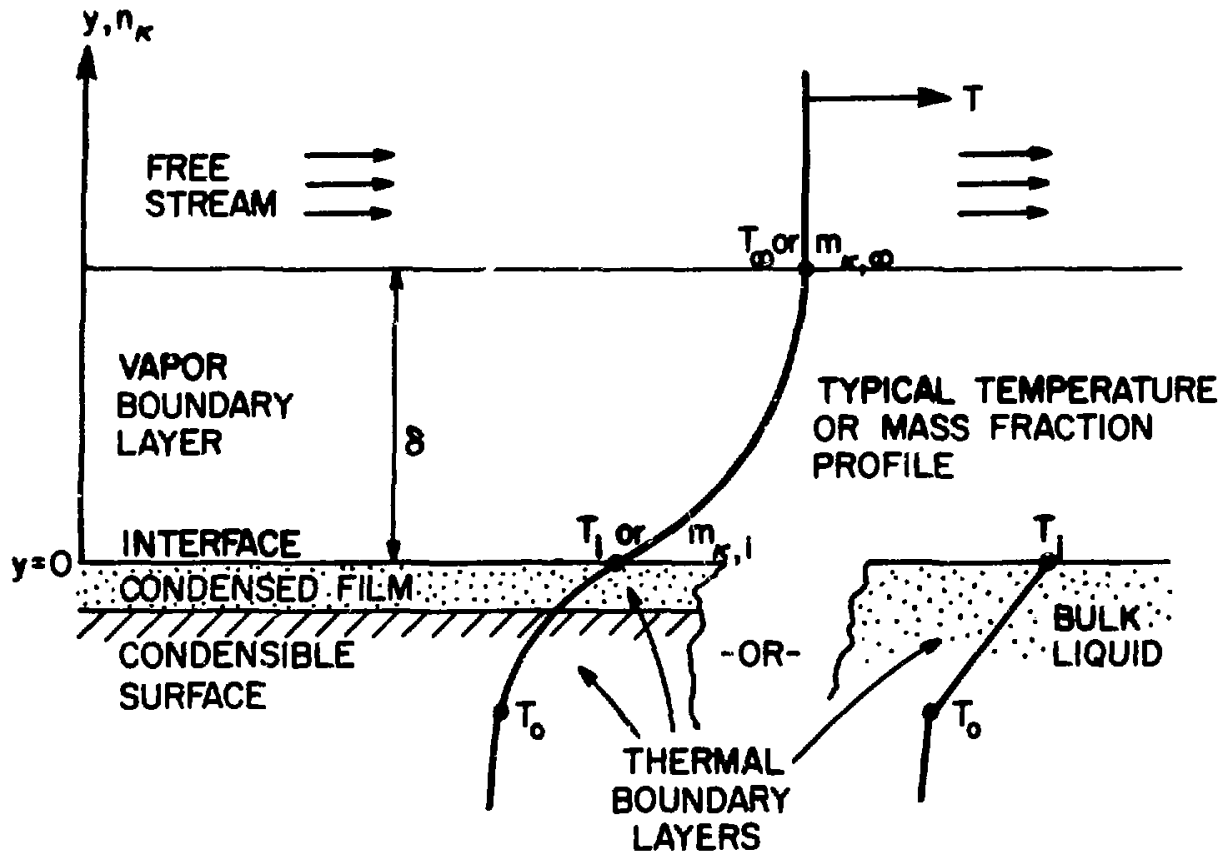

Fig. 1.

The heat and mass transfer model.

thickness $\delta$ can be obtained either from a solution to the boundary layer equations or from a mass transfer coefficient.

The mass flux boundary condition is found simply by evaluating Eq. (1) at the interface $i$. Various modes of coupling exist in a system composed of $N$ species. A direct linear mode of coupling exists through the convective term $\rho v$. The convective term is the net mass flux through the boundary layer, which in turn is the sum of all the individual mass fluxes. Hence an $N$ component system is governed by a matrix equation of the form

$$
n_{K}=\left(\sum_{\lambda=1}^{N} n_{\lambda}\right) m_{\kappa, i}-\left.\rho D_{K, g} \frac{\partial m_{K}}{\partial y}\right|_{y=i}, \quad k=1 \ldots N \text {. }
$$

There are two other modes of coupling. First, the net flow through the boundary layer will have an influence upon the mass fraction gradient. This form of coupling is nonlinear in nature and will be discussed further in the section entitled "The Correction Factor". The last mode of coupling is through the thermodynamic behavior of the materials in the condensed phase. The vapor mass fraction of species $k$ at the vapor-liquid interface is governed 
primarily by the temperature of the interface. This dependence means that the energy equation must also be included in the analysis.

The enthalpy flux is found by integrating the evergy equation and extracting that portion which represents the flux:

$$
\text { enthalpy flux }=-k \frac{\partial T}{\partial y}+\rho v h-\sum_{K=1}^{N} \rho D \frac{\partial m_{K}}{\partial y} h_{K}
$$

The terms $-k \frac{\partial T}{\partial y}$, $\rho v h$ and $\sum_{k=1}^{N} \rho D \frac{\partial m_{\kappa}}{\partial y} h_{k}$ are thermal heat conduction, bulk convection of enthalpy, and diffusion of enthalpy. Enthalpy $h$ is based upon mass weighting so that the bulk convection term is composed of the bulk convective mass flux times the individual species enthalpies weighted by their respective mass fractions; that is,

$$
\rho v h=\rho v \sum_{\kappa=1}^{N} h_{K} m_{K}
$$

Combining Eqs. (2), (3), and (4) yields

$$
\text { enthalpy flux }=-k \frac{\partial T}{\partial y}+\sum_{K=1}^{N} n_{K} h_{K} \text {. }
$$

The phase transition interface cannot store energy or mass, hence enthalpy and mass fluxes entering and leaving must be equal. Equating the fluxes at points above (subscript a) and below (subscript b) the interface results in

$$
\sum_{K=1}^{N} n_{K}\left(h_{K, a}-h_{K, b}\right)=+\left.k_{a} \frac{\partial T}{\partial y}\right|_{a}-\left.k_{b} \frac{\partial T}{\partial y}\right|_{b}
$$


The enthalpy difference between points $a$ and $b$ is the latent heat of vaporization $h_{\ell g}$ (or sublimation as the case may be). Overall heat transfer coefficients can be substituted for the gradients of temperature; hence, the final energy flux boundary condition equation is

$$
\sum_{K=1}^{W} n_{K} h_{l g, K}=H_{g}\left(T_{\infty}-T_{i}\right)+H_{0}\left(T_{0}-T_{j}\right),
$$

where

$H$ is an overall heat transfer coefficient.

The subscripts $g$ and $o$ refer to vapor phase and condensed phase, respective1y. The condensed phase heat transfer coefficient, $\mathrm{H}_{0}$, will include the condensed film, if it exists, and any other layers of material which may exist between the liquid vapor interface ( $i$ ) and the condensed phase reservoir ( 0 ). If a situation involving time or position dependence or both exists, then the heat cransfer coefficients $H_{g}$ and $H_{0}$ and the temperatures $T_{\infty}, T_{i}$ and $T_{0}$ will be functions of time or position or both.

B. Thermodynamic Equilibrium of the Interface

In this section, relationships giving the mass fraction of species $k$ as a function of the interface temperature and component miscibility will be derived. The ininererit assumption is that the multicomponent vapors adjacent to the interface are in saturated thermodynamic equilibrium with the liquid (or solid) condensed phase at the system total pressure $P$. The error introduced by making this assumption is negligible if the temperature gradient at the interface is small over the mean free path distance of the molecules. Possible exceptions are explosive vapor formation or condensation of highly supersaturated vapors. Although explosive vapor formation can occur in some instances (for example, fuel-coolant interactions) other unknown factors such as the surface area for vapor formation will introduce more error than the assumption of thermodynamic equilibrium. Recent experimental investigations indicate that alkali metal condensation at low pressure may involve nonequilibrium thermodynamics. ${ }^{2}$ This question has not yet been fully resolved because there is considerable scatter in the data and, in addition, other 
phenomena such as molecular dissassociation, which is akin to chemical reactions, can be occurring at the interface. Further, pressure gradients normal to the interface cannot be ignored at low pressures.

The vapor pressures of the various species depend to a large extent upon the miscibility of the various components. Rather than getting deeply i. polved in the thermodynamic behavior of mu?ticomponent mixtures, two simple inidels can be used. For immiscible systems each component will be assumed to exert its saturation vapor pressure independent of its concentration in the condensed phase. ${ }^{3}$ For a completely miscible system each component will be assumed to exert a vapor pressure equal to its saturation pressure multiplied by its mole fraction in the condensed phase, this is sometimes called Raoult's Law.

A simplified relationship for the saturation vapor pressure of a component $\left(P_{K}\right)$ as a function of surface temperature $\left(T_{S}\right)$ can be obtained by

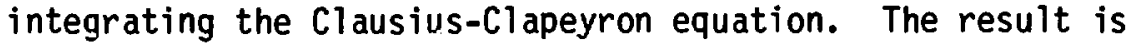

$$
P_{K}=P_{K}^{*} e^{-T_{K}^{*} / T_{S}}
$$

The parameters $P_{K}^{*}$ and $T_{K}^{*}$ were held constant during this integration and can be evaluated bi curve fits to the $\kappa^{\text {th }}$ species saturation vapor pressure curve. The curve fits obtained by using $\mathrm{q}$. (8) are found to be quite reasonable, yielding vapor pressures accurate to within 10-30\% over a range from subatmospheric to the critical point for many substances.

The mass fraction of species $k$ is defined as the ratio of partial density $\left(\rho_{K}\right)$ to the total density $\rho$. By definition, the partial density of a given species is proportional to the product of molecular weight and vapor mole fraction. From the ideal gas law, the vapor mole fraction is equal to the ratio of partial pressure of species $\kappa$ to the total pressure $P$. Thus an expression for the mass fraction of species $\kappa$ can be written in terms of molecular weights and pressures:

$$
m_{K}=\frac{\rho_{K}}{\rho}=\frac{M_{K} P_{K} / P}{\sum_{K} M_{K} P_{K} / P}=\frac{M_{K} P_{K}}{\sum_{K} M_{K} P_{K}},
$$


where

$$
\begin{aligned}
& M_{K}=\text { molecular weight of species } \kappa, \\
& F_{K}=\text { partial pressure of species } \kappa .
\end{aligned}
$$

Evaluation of the mass fraction in the vapor free stream $\left(m_{k, \infty}\right)$ is usually a simple matter because the composition of the vapor and the partial pressures of the various components are known or are given conditions.

Evaluation of the mass fractions of the various species at the interface. is not necessarily straightforward because numerous possibilities exist. For example, there may or may not be noncondensibles present, or conditions may be such that a given species can be condensible in one case and act as a noncondensible gas in another. This latter condition is usually determined by a trial and error procedure to be described later.

Evaluating the mass fractions of the various species at the surface relies upon the assumption of constant pressure through the boundary layer normal to the interface. This assumption introduces negligible error because the vapor must have an appreciable kinetic energy in order to effect the total pressure at the interface. Explosive vapor formation and condensation of alkali metals at low pressure may be possible exceptions to this assumption.

Since the condensed phase at the interface is in saturated thermodynamic equilibrium with the vapor and the pressure of the interiace is known, then from thermodynamics the interface temperature is also known provided that noncondensible gases do not exist. The surface temperature in the case of no noncondensible gases is the boiling point of the multicomponent mixture.

If the multicomponent mixture is completely immiscible then the boiling point is called the eutectic temperature. The eutectic temperature is the point at which the sum of the partial pressures equals the total pressure, i.e.,

$$
P=\sum_{K=1}^{N} P_{k}=\sum_{K=1}^{N} P_{k}^{*} e^{-T_{K}^{*} / T S} .
$$

The surface temperature in this case is found by solving Eq. (10) for

$$
\text { Ts. }
$$


If the multicomponent mixture is miscible then the surface temperature is found by inverting an analogous equation for miscible systemis, that is,

$$
P=\sum_{K=1}^{I} P_{K}=\sum_{K=1}^{I} X_{K} P_{K}^{*} e^{-T_{K}^{*} / T_{S}},
$$

where

$x_{K}$ is the mole fraction of the species $k$ in the condensed phase.

Note the difference in the formulas. The surface temperature of the immiscible system is independent of the concentration of the various species in the condensed phase.

If noncondensible gases do exist in the problem under investigation, then the total pressure at the interface is composed of the saturation pressures of the various species in the condensed phase plus the partial pressure of the nonconder,sible gas. Hence $P$ in Ẹ̃s. (12) and (13) should be replaced with $P-P_{n c, i}$, where $P_{n c, i}$ is the partial pressure of the noncondensible gas at the interface. Unfortunately, the $P_{n c, i}$ is unknown a priori and is part of the solution to the problem. Numerically and, possibly, conceptually it is easier to work with an unknown surface temperature $T_{j}$ rather than an unknown noncondensible partial pressure $P_{n c, i}$, although the formulations are entirely equivalent.

C. The Mass Transfer Coefficient

In order to generate a solution to Eqs. (2) and (7) together with the conditions given in Sec. II.B it is best to simplify Eq. (2) even further by introducing a mass transfer coefficient $G$. The reason for doing this is to allow the theory to be applicable to all flow systems and geometries for which heat transfer coefficients are available.

The mass transfer coefficient is defined in a manner analogous to the heat transfer coefficient. Recall that the heat transfer coefficient is defined as

$$
-\left.k_{g} \frac{\partial T}{\partial y}\right|_{i}=-H_{g}\left(T_{\alpha}-T_{j}\right)
$$


By analogy, one can define a mass transfer coefficient as

$$
-\left.\rho D_{k, g} \frac{\partial m_{k}}{\partial y}\right|_{i}=-G_{k}\left(m_{k, \infty}-m_{k, i}\right) \text {, }
$$

where

$$
\begin{aligned}
& D_{k, g} \quad \text { is the mass diffusivity of species } k \text { through the } \\
& G_{K} \quad \text { is the mass transfer coefficient of species } k \text {, } \\
& m_{k, \infty} \text { and } m_{K}, i \quad \text { are the mass fractions of species } k \text { in the yapor free }
\end{aligned}
$$

In order to derive a mass transfer coefficient from a heat transfer coefficient, one relies upon the similarity of the boundary layer equations for energy and mass flow. The thermal diffusivity and the mass diffusivity appear in the same location in both equations. Heat transfer correlations presumably contain an approximate solution to the boundary layer equations in terms of an equivalent stagnant boundary layer thickness $\delta$.

The simplest method of generating an approximate mass transfer coefficient is to use the Lewis number equal unity simplification. The Lewis number is the ratio of Schmidt to Prandtl number, and by setting it equal to unity one is equating the mass and thermal diffusivities.

By using the unit Lewis number simplification, the boundary layer equations are identical, and hence the equivalent stagnant boundary layer thicknesses are identical. Thus equating the thermal and species stagnant boundary layer thicknesses results in

$$
\delta_{t h}=\frac{K}{H}=\delta_{m}=\frac{\rho D}{G} \text {. }
$$

Rearranging

$$
G^{*}=\frac{G D H}{k}
$$


and using the unit Lewis number simplification $\left(D=\frac{k}{\rho C}\right)$ results in

$$
G^{\star}=\frac{H}{C_{p}}
$$

The * superscript upon $G$ denotes that the mass transfer coefficient is valid only at very small mass transfer rates.

This level of approximation, the Lewis number unity simplification, turns out to be reasonably good for gases but quite poor for 1 iquids because liquids usually have very large Lewis numbers.

The next higher level of approximation is to use the actual binary diffusion coefficients of the various species. For a two component system, the mass transfer coefficient $G^{\star}$ is

$$
\mathrm{G}^{*}=\rho \mathrm{Q}_{12} \mathrm{Nu} / \mathrm{L}
$$

where $\mathrm{Nu}$ is the Nusselt number and $\mathrm{L}$ is the characteristic length in the Nusselt number corre? ation.

If three or more components exist, then an additiona? nonlinearity is introduced due to the requirement that the sum of all the diffusive fluxes must be zero. To satisfy this requirement, the multicomponent mass diffusivities $D_{K, g}$ will depend upon the binary diffusion coefficients for each pair of components as well as upon all of the mass fluxes. The dependence can be obtained by equating the expression for the absolute mass flux of species $k$ to the Stephan-Maxwell formulation for multicomponent diffusion. The result is 1

$$
D_{k, g}=\frac{\rho\left(n_{k}-m_{k, i} \sum_{\lambda=1}^{N} n_{\lambda}\right)}{\sum_{\lambda=1}^{N} \frac{1}{\rho D_{k, \lambda}}\left(m_{\lambda, i} n_{k}-m_{k, i} n_{\lambda}\right)} .
$$

Equation (18) can be used wherever the mass diffusivity of species $\kappa$ in the gas mixture $g$ appears. The use of this formulation adds additional nonlinearity to the system of equations. Engineering judgment shourd be used to 
decide whether or not a more accurate solution, utilizing the multicomponent mass diffusivity, is desired. Numerical experience indicates that the inclusion of this formulation could lead to differences in prediction as large as a factor of two or more, depending upon the problem under analysis. D. The Correction Factor

As stated in earlier sectiors, the mass transfer coefficient $G^{*}$, which is obtained from heat transfer data, is only valid in the limit of no mass transfer; the reason is that the normal component of velocity is nonzero at the surface whenever there is a finite mass transfer rate. In this section, a correction factor will be derived which when multiplied by $G{ }^{*}$ yields an appropriate mass trarsfer coefficient $G$ that has been corrected for the nonzero component of velocity at the surface.

The starting point for this derivation is the Couette flow boundary layer approximation. In the couette flow approximation, derivatives in the direction parallel to the surface are neqlected in comparison with derivatives normal to the surface. Thus the species equation at steady state reduces to

$$
D_{k, g} \frac{d^{2} m_{k}}{d y^{2}}-v \frac{d m_{k}}{d y}=0
$$

This equation is integrated twice and the boundary conditions

$$
\begin{array}{ll}
m_{K}=m_{k, i} & \text { o } y=0 \\
m_{K}=m_{k, \infty} & \text { o } y=\delta
\end{array}
$$

are used to solve for the constants of integration. The resulting solution is

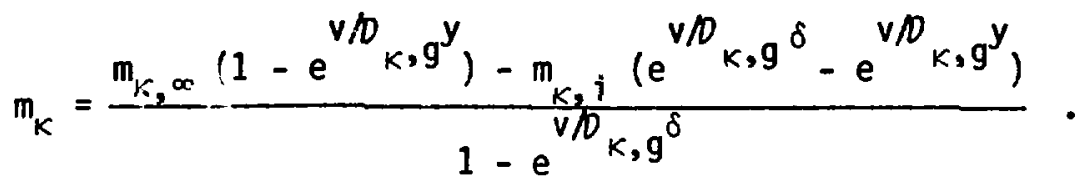

By analogy to the heat transfer situation a mass transfer coefficient is sought which yields the diffusive component of the mass flux 
$-\left.\rho D_{K, g} \frac{\partial m_{K}}{\partial y}\right|_{y=0}=-G_{K}\left(m_{K, \infty}-m_{K, i}\right)=-G_{K}^{*}\left(\frac{G}{G^{*}}\right)_{K}\left(m_{K, \infty}-m_{K, i}\right)$.

The term $\left(\frac{G}{G^{\star}}\right)$ is introduced as a correction factor which when multiplied by $G^{\star}$, yields a mass transfer coefficient $G$ that has been modified to account for a finite mass transfer rate. The correction factor can be found either by solving Eq. (22) for $\left(\frac{G}{G^{\hbar}}\right)$ or by evaluating the ratio;

$$
\left(\frac{G}{G^{\star}}\right)_{K}=\frac{\frac{\partial m_{K}}{\partial y} y=0}{\left.\operatorname{limit}_{y \rightarrow 0} \frac{\partial m_{K}}{\partial y}\right|_{y=0}} .
$$

Differentiating Eq. (21) and substituting into Eq. (23) yields

$$
\left(\frac{G}{G^{\star}}\right)_{\kappa}=\frac{\frac{v \delta}{D_{K, g}}}{e^{\frac{v \delta}{D_{K, g}}-1}} .
$$

From the definition of a mass transfer Nusselt number, we have

$$
\delta=\frac{L}{N u}=\frac{P D_{K, g}}{G_{K}^{\star}} \text {, }
$$

where

$L$ is the characteristic length scale of the Nusselt number correlation.

Hence the corrected mass transfer coefficient is 


$$
G_{K}=G_{K}^{*}\left(\frac{G}{G^{*}}\right)_{K}=G_{K}^{*} \frac{\left(\sum_{K=1}^{N} n_{K}\right) / G_{K}^{*}}{\left(\sum_{K=1}^{N} n_{K}\right) / G_{K}^{*}} .
$$

Note that the mass transfer coefficient is species dependent and can be used in this manner provided that the appropriate species mass diffusivity $D_{K, g}$ is defined for the multicomponent mixture [Eq. (18)]. Alternatively one could use the unit Lewis number assumption, in which case the mass transfer coefficient is not species dependent and $G_{K}^{\star}$ reduces to $G^{\star}=$ $H_{g} / C_{p}$

The energy equation also has a correction factor that can be derived in the same manner. If the mass diffusivity 0 is replaced by thermal diffusivity $\alpha$, mass fractions by temperatures, and mass transfer coefficients by heat transfer coefficients, then a corrected heat transfer ;oefficient is found to be

$$
H_{g}=\frac{\left(\sum_{K=1}^{N} n_{K} c_{p, \kappa}\right)}{e^{\left(\sum_{K=1}^{N} C_{p, \kappa^{n}}{ } / H_{g}^{*}\right)_{-1}}}
$$

For the case Le $=1$ the correction factors

$$
\frac{H_{g}}{H_{g}^{*}} \text { or } \frac{G}{G^{\star}}
$$

are identical. From Eqs. (25) and (26) the corrected heat and mass transfer coefficients reduce to starred values when there is no net mass flux (that is, $\sum_{K=1}^{N} n_{K}=0$ ). If the net mass flux is negative (net condensation) then the heat and mass transfer coefficients are enhanced. The reverse effect occurs when the net mass flux is evaporative. 


\section{E. Solution Techniques for Simultaneous Heat and Mass Transfer}

Summarizing, the equations to be solved are $\mathbf{N}$ species equations of the form

$$
n_{k}=\sum_{\lambda=1}^{N} n_{\lambda} m_{i, k}-G_{k}\left(m_{\infty, k}-m_{i, k^{\prime}},\right.
$$

and an energy equation

$$
\sum_{\kappa=1}^{N} n_{\kappa} h_{\ell g, K}=H_{g}\left(T_{\infty}-T_{i}\right)+H_{0}\left(T_{0}-T_{j}\right) .
$$

The species and energy equations are coupled at the interface by a thermodynamic condition

$$
m_{i, k}=\frac{M_{K} P_{i,}}{\sum_{K=1}^{N} M_{K} P_{i, k}}, P_{i, K}=P_{K}^{*} e^{-T_{K}^{*} / T_{i}} \text { (immiscible systems) . }
$$

If noncondensible gases are present then the partial pressure $P_{i, K}$ of the noncondensible gas is unknown. The heat and mass transfer coefficients are coupled to the mass fluxes by the relations

and

$$
G_{K}=\frac{\left(\sum_{k=1}^{N} n_{K}\right)}{e^{\left(\sum_{k=1}^{N} n_{K}\right) / G_{K}^{*}-1}},
$$

$$
H_{g}=\frac{\sum_{k=1}^{N} n_{k} c_{p, k}}{e^{\left(\sum_{k=1}^{N} n_{k} c_{p, k}\right) / H_{g}^{*}}},
$$


and finall:' the starred heat and mass transfer coefficients are found from experimental correlations of which

$$
N u_{H}=\frac{H_{g}^{*} L}{k}=f(R e, P r),
$$

and

$$
N u_{m}=\frac{G_{K}^{\star} L}{\rho_{g} D_{K, g}}=f(R e, S c) \text {, }
$$

are typical examples. Nu, Re, Pr, and Sc are the Nusselt, Reynolds, Prandtl, and Schmidt numbers, respectively.

The solution technique for this highly coupled nonlinear set of equations will be presented subsequently for the case of an immiscible fluid system with a unit Lewis number for all species. The method will be presented in a stepwise fashion for clarity, and each step may contain a set of parallel or sequential operations depending upon the various possibilities that exist.

Step 1. Calculate all of the variabies which do not change during the iteration such as the free stream mass fractions $m_{k, \infty}$ and the eutectic temperature of the multicomponent mixture. Initial guesses are made of the starred heat and mass transfer coefficients $\mathrm{H}_{\mathrm{g}}^{*}, \mathrm{G}^{*}$ anid $\mathrm{H}_{0}$. These are considered initial guesses because property variations due to concentration and temperature gradients can affect their values. The condensed phase heat transfer coefficient $H_{0}$ is aiso an initial guess because any liquid film that is present is a function of the concensing mass fluxes $i$.

Step 2. In this step a decision is made determining whether noncondensible gases are present and if so which species. If it cannot be determined whather a particular species is noncondensible, it can be ass"... to be condensible, and this assumption can be checked for consistency in Step 7.

Case $i$. No noncondensible gases. In this case the interface temperature is known to be the eutectic temperature of the multicomponent mixture. The vapor mass fractions $m_{K}, i$, latent heats of vaporization $h_{\ell g}$, and thermophysical properties of the vapor are all evaluated at the interf ace temperature.

Case II. Noncondensible gases present. In this case the interface temperature is not known so that an initial guess is made. A simple methed of 
solving for the surface temperature is to start at just under the upper limit, (that is, the eutectic temperature) and increment downwards until a furctiona1, defined in Step 6, changes sign. Then use a Newton-Raphson technique to converge upon the surface temperature. With each guess of the surface temperature the corresponding mass fractions, latent heats, and thermophysical properties are evaluated.

Step 3. Set up and solve the matrix equation. The matrix Eq. (27) becomes a singular equation if the sum of the surface mass fractions $m_{K, i}$ equals unity. The singularity is due to an overspecification of the mass transfer problem and can be overcome by replacing one of the species equations with the energy equation.

Case I. No noncondensible gases. In this case, any of the species equations can be replaced by the energy equation; hence, the system of equations to be solved becomes

$$
\begin{aligned}
& n_{K}-\sum_{\lambda=1}^{N} n_{\lambda} m_{K, i}=G^{\star}\left(\frac{G}{G^{\star}}\right)\left(m_{K, \infty}-m_{K, i}\right), \quad K=1, N-1, \\
& \sum_{K=1}^{N} h_{\ell g, K} n_{K}=H_{g}^{*}\left(\frac{G}{G^{\star}}\right)\left(T_{\infty}-T_{i}\right)+H_{0}\left(T_{0}-T_{i}\right) .
\end{aligned}
$$

Case II. Noncondensible gases present. In this case one of the species fluxes is known at the outset; that is, the flux of noncondensible gas is zero by definition. However, the surface temperature in this case is unknown so that one unknown has been replaced by another. Thus the species matrix equation reduces to

$$
n_{K^{*}}-\sum_{\lambda=1}^{N-1} n_{\lambda} m_{K, i}=G^{*}\left(\frac{G}{G^{*}}\right)\left(m_{K, \infty}-m_{K, j}\right), \quad k=1, N-1 .
$$

Note that on $1 y^{\prime}$ N-1 equations are invoived. The Nth equation is the noncondensible gas mixture equation. The interface mass fractions $m_{\lambda_{i}}$ are based upon the guessed surface temperature $T_{i}$. The species mass fluxes $n_{k}$ that 
result from solving this system of equations do not necessarily satisfy the energy equation, which in turn leads to a method whereby the interface temperature can be updated to an interface temperature which, does satisfy the energy equation.

Step 4. Solve for the correction factor $\left(\frac{G}{G^{\star}}\right)$.

Case I. No noncondensible gases. The correction factor

$\left(\frac{G}{G}\right)$ can be facto ed out of the resultant vector in Eq. (33). Factoring,

solving the linear system of equations, and summing results in

$$
\sum_{k=1}^{N} n_{K}=\left(\frac{G}{G^{\hbar}}\right) \underline{A}+\underline{B} .
$$

where

$\underline{A}$ and $\underline{B}$ are sums over the resultant vectors on the right hand side Substituting for $\left(\frac{G}{G}\right)$ from Eq. (25) results in

$$
\sum_{k=1}^{N} n_{K}=\frac{\underline{A} \sum_{K=1}^{N} n_{K}}{\left(\sum_{K=1}^{N} n_{K} / G^{*}\right)_{-1}}+
$$

This nonlinear equation can be solved by a Newton-Raphson technique for the net mass flux $\sum_{K=1}^{N} n_{K}$.

Case II. Noncondensible gases present. This case is handled in the same manner as Case I with the exception that a Newton-Paphson technique is not required because the vector sum $\underline{B}$ does not appear. Ine nonlinear equation can be inverted to yield the correction factor 


$$
\frac{G}{G^{*}}=\frac{\ln \left(1+A / g^{*}\right)}{\underline{A} / g^{*}} \text {. }
$$

Step 5. Convergence check.

Case I. No noncondensible gases. The only variables to be converged are the net mass flux in the Newton-Raphson iteration of Step 4 Case I and the heat transfer coefficient $H_{g}$ and $H_{0}$ (if they are variables). If convergence is obtained to some desired degree of accuracy, proceed to Step 7; if not, proceed to Step 6 .

Case II. Noncondensible gases present. In this case the surface temperature needs to satisfy both the species and the energy equation. If the mass fluxes resulting from Step 3, Case II and Step 4, Case II are substituted into the energy equation, an interface temperature can be predicted:

$$
T_{i}^{\prime}=\frac{H_{g}^{*}\left(\frac{G}{G^{\star}}\right) T_{\infty}+H_{0} T_{0}-\sum_{K=1}^{N} n_{K} H_{\ell g, K}}{H_{g}^{\star}\left(\frac{G}{G^{\star}}\right)+H_{0}} .
$$

The prime indicates that this interface temperature does not necessarily equal the guessed interface temperature from Step 2, Case II. If $T_{\mathfrak{j}}^{\prime}$ does equal $T_{\mathfrak{j}}$ withir, some specified degree of error, proceed to Step 7 ; if not proceed to Step 6.

Step 6. Update changing variables.

Case I. No noncondensible gases. The only variables that can change in this case are the heat and mass transfer coefficients because they can depend upon the magnitude and sign of the mass of fluxes $n_{K^{*}}$ Return to Step 3.

Case II. Noncondensible gases present. In this case, the interface temperature, the vapor-side and the liquid side heat and mass transfer coefficients are updated. The interface temperature can be updated by defining a' variable

$$
F=T_{i}-T_{i}^{\prime} \text {. }
$$


If $T_{i}$ is reduced from the eutectic temperature in a stepwise fashion, the variable $F$ will change sign when the correct interface temperature has been straddled. A Newton-Raphson technique can then be used to locate the point at which $F=0$, using numerical derivatives of $F$ with respect to $T_{i}$. The vapor heat and mass transfer coefficients can be updated according to the average thermophysical properties of the vapor in the boundary layer. $H_{0}$ can be updated according to the mass fluxes $n_{K}$, provided a dependency exists as in condensed liquid films. Return to step 3.

Step 7 . In this step the initial assumptions made at the beginning of the iteration are checked for consistency. The initial assumptions referred to here are the partial vapor pressures of the condensed phase species. At the beginning of the iteration (Step 2) it was assumed that all species that exist in the vapor phase also exist in the conciensed phase with the exception of true noncondensible gases (such as air, noble gases, etc.). This need not be the case, however, because a condensible species can also act as a noncondensible. For example, consider simultaneous condensation of sodium, steel, and $\mathrm{UO}_{2}$ vapors upon cold steel structures in the absence of fission gas. If the steel structure is cold enough then all three species will condense. However, if the structure temperature is too high, sodium will not condense. In fact, if a thin film of sodium were present upon the structure it would evaporate simultaneously as the others condensed. The evaporation-condensation transition point cannot be predicted at the outset; hence, this step is required as a check to see if the evaporative fluxes ( $n_{k}$ positive) are consistent with that particular species existing in the condensed phase. If it is not consistent, then the inconsistent species must be treated as a noncondensible gas and the calculations are repeated, that is, return to Step 2. If all the fluxes are consistent, then one has a solution.

\section{MODELING CONCERNS}

Phenomena that can be important in phase transitions and were only mentioned briefly or ignored altogether will be discussed in this section. It will be pointed out how various phenomena are included or how the model may be modified to include them. The phenomena can be grouped into five categories; film physics, transient effects, nonequilibrium effects, thermodynamic effects, and thermal radiation. 
Film physics can be very important whenever condensation, or absorbtion, is occurring Films can exert control over a condensation process through either a heat or mass transfer mechanism.

Film control by heat transfer can be included in the model by using an overall heat transfer coefficient $H_{0}$ (Eq. (7)) that includes both the condensed $f i l m$ heat transfer resistance and the condenser resistance.

Film control by mass transfer can occur when a miscible system of components exists or when crystallization is occurring. Under these conditions, the vapor mass fractions at the interface are not a simple function of temperature as they are with an immiscible system of components. An additional set of equations which describes the diffusion and convection of components at the interiace into the bulk liquid phase or through the porous crystallized film is required. These equations would be used in determining the mole or mass fractions of the various components at the interface. Equation (11) can then be used to solve for the corresponding vapor mass fractions for miscible liquid systems.

There are three methods of including transient effects into the model. The simplest methods are to use time dependent transfer coefficients and quasistatic methods. If these methods prove inadequate, then solutions to time dependent boundary layer equations must be used.

Time dependent transfer coefficients can be used if the problem under analysis is similar to one where an exact analytic transient solution exists. In this case, the applicable time dependent heat transfer coefficients would be used in Eq. (7).

Quasistatic methods are applicable if the transient effect of building up or changing a boundary layer is negligible over the time scale of interest. Under these conditions, steady state correlations based upon transient free stream and bulk conditions are used to obtain the heat transfer coefficients.

Solving the time dependent boundary layer equations will lead to solutions of greater accuracy if desired. A simpler method of doing this would be to solve the time dependent Couette boundary layer equation. (The time dependent version of Eq. (19).) The solution should include time derivatives of the boundary layer thickness $\delta$ and free stream conditions if they are changing. If this approach is used there is no need to define heat or mass 
transfer coefficients since the interface gradients $\left.\kappa \frac{\partial T}{\partial y}\right|_{y=0}$ and $\left.D \frac{\partial m_{K}}{\partial y}\right|_{y=0}$ can be used directly in obtaining solutions to the problem. Lastly, if the Couette boundary layer equations prove inadequate then the full nonlinear boundary layer equations can be used.

Nonequilibrium effects can exist during a phase transition process. Superheat and supersaturation are included automatically in the model through tni, jefinition of the free stream vapor temperature and mass fractions. These effects pose no problem with the exception of instabilities that can arise with extreme supersaturation. In an extremely supersaturated state, if the sensible heat of cooling $\left(C_{j}\left(T_{i}-T_{\infty}\right)\right)$ exceeds the latent heat of evaporation $h_{\ell g^{\circ}}$ a steady solution is not possible. In practice, this situation should not occur since spontaneous nucleation will occur long before a highly supersaturated state could be created. Spontaneous nucleation and condensation within the boundary layer can occur under some circumstances. This effect has not been included in the model. There is as yet no experimental evidence to indicate how important this phenomenon is.

Thermodynamic nonequilibrium of the interface has received considerable attention in the literature. ${ }^{4}$ These effects can be included in the model by modifying the relationships in Sec. II.B to include a dependence upon the mass fluxes and the "condensation coefficient."

Thermodynamic effects can play a role when critical point conditions are encountered duri $g$ the phase transition process. An example is condensation of hot $\mathrm{UO}_{2}$ vapor at high pressure upon cold sodium. If the pressure exceeds the critical pressure of sodium then the interface temperature can exceed the critical temperature of sodium. What phenomena control the rate of sodium vaporization under these severe conditions is not clear, and consequently such physics has not been included in the model. Experimental data with other materials may help to determine what phenomena are important under these conditions.

Thermal radiation can be included in the model by adding an additional term to Eq. (7), which accounts for the radiative heat flux to the interface. There are other complex phenomena which can occur during a multicomponent phase transition process; one must use engineering judgment to determine if a particular phenomenon is controlling, and if so how to include it into the model. 


\section{SUMMARY AND CONCLUSIONS}

This report introduces the reader to an approximate technique for solving the multicomponent simultaneous heat and mass transfer problem. The method is sufficiently general to include most of the effects that are normally encountered in multicomponent phase transitions. The method is structured such that any of the simplifying assumptions need not be made and can be improved upon without changing the method but only increasing the nonlinearity of the system of equations.

A future report will concentrate upon an experimental verification of the method and an assessment of the accuracy of the various assumptions.

\section{REFERENCES}

1. B. R. Bird, W. E. Stewart, and E. N. Lightfoot, "Transport Phenomena," (John Wiley \& Sons, Inc., New York, 1960) p. 571.

2. S. Necmi and J. W. Rose, "Film Condensation of Mercury," Int. J. of Heat and Mass Transfer, Vol. 19, pp. 1245-1256 (1976).

3. 0. A. Hougen, K. M. Watson, R. A. Ragatz, "Chemicái Process Principles," (John Wiley \& Sons, Inc., New York, 1943) pp. 97-99.

4. R. W. Schrage, "A Theoretical Study of Interphase Mass Transfer," (Columbia University Press, New York, 1953). 